\title{
Analysis of 5-hydroxytryptamine and its related indoles in cerebrospinal fluid of leukemic children by gas chromatography-mass spectrometry
}

https://doi.org/10.1515/labmed-2019-0156

Received September 18, 2019; accepted December 27, 2019; previously published online January 28, 2020

\section{Abstract}

Background: It is meaningful to quantify some neurotransmitters in cerebrospinal fluid (CSF) for pathology and clinical diagnosis. The objective of this study was to develop a rapid and reliable method for detecting 5-hydroxyindole ethanol (5-HTOL), 5-hydroxyindole acetic acid (5-HIAA), 5-hydroxytryptophan (5-HTP) and 5-hydroxytryptamine (5-HT) in CSF by gas chromatography-mass spectrometry (GC-MS), and explore the clinical significance of the levels of these neurotransmitters in CSF from acute lymphoblastic leukemia (ALL).

Methods: The levels of 5-HTOL, 5-HIAA, 5-HTP and 5-HT in CSF from children with ALL and a control (CON) group were examined by the proposed GC-MS method.

Results: The GC-MS method showed good sensitivity and accuracy. 5-HT and 5-HIAA contents in the ALL group were significantly lower than those in the CON group $(\mathrm{p}<0.01)$, while there was no significant difference in 5-HTP and 5-HTOL contents between the two groups ( $p>0.05)$.

Conclusions: This sensitive and reliable method could be used in studies focusing on clinical pathogenesis of ALL.

Keywords: 5-hydroxyindole acetic acid; 5-hydroxytryptamine; cerebrospinal fluid; gas chromatographymass spectrometry; leukemia.

aPingping Zhang and Bo Wang contributed equally to this work. ${ }^{*}$ Correspondence: Kaoqi Lian, School of Public Health, Hebei Medical University, Shijiazhuang, P.R. China; and Hebei Key Laboratory of Environment and Human Health, Shijiazhuang 050017, P.R. China, Phone: +(86)-311-8626 1043, Fax: +(86)-311-8626 5754 E-Mail: liankq@hebmu.edu.cn

Pingping Zhang, Yanmei Sun and Jian Gao: Department of Reproductive Genetic Family, Hebei General Hospital, Shijiazhuang, P.R. China

Bo Wang: Handan College, Handan, P.R. China

¿ Open Access. ( 2020 Kaoqi Lian et al., published by De Gruyter. (c) BY Public License.

\section{Introduction}

5-Hydroxytryptamine (5-HT), also known as serotonin, is an indole derivative that is ubiquitous in mammals. As an inhibitory neurotransmitter, 5-HT is highly expressed in the cerebral cortex and nerve synapses [1]. It is a tryptophan metabolite and is further catalyzed mainly by monoamine oxidase into 5-hydroxyindole acetic acid (5-HIAA) or 5-hydroxyindole-3-ethanol (5-HTOL) via 5-hydroxyindole acetaldehyde. 5-HT and related indole derivatives possess multiple and complex biological activities [2]. They are involved in several systemic physiological and pathological responses and are closely associated with many diseases such as tumors and neuropsychiatric disorders $[3,4]$.

Leukemia develops from malignant clones of hematopoietic stem cells. Leukemic cells exhibit typical tumor cell characteristics such as over-proliferation, blocked differentiation and apoptosis. These cells can propagate and aggregate in the bone marrow and other hematopoietic tissues, resulting in inhibition of normal hematopoiesis and immune functions. Leukemic cells gradually replace normal hematopoiesis and infiltrate into the liver, spleen, lymph node, central nervous system and skeletal tissues [5]. The cerebrospinal fluid (CSF), produced and filtered from the choroid plexuses of cerebral ventricles, spreads over the surfaces of the brain and spinal cord upon entering the subarachnoid space and returns to the dural venous sinuses and circulation via the arachnoidal granulations [6]. Thus, analysis of the constitution of the CSF is important in the studies of related diseases. In addition, altered concentrations of 5-HT and related indole derivatives in the CSF are implicated in several neurological disorders [7]. Hence, accurate determination of 5-HT is important to elucidate the physiological and pathological roles of 5-HT as well as for the prevention, diagnosis and treatment of related diseases. Therefore, on the basis of previous research [8], we attempted to use this method for the analysis of 5-HT, 5-hydroxytryptophan (5-HTP), 5-HIAA and 5-HTOL in the CSF of children with leukemia. 


\section{Materials and methods}

5-Hydroxytryptamine (5-HT) was purchased from Sigma (St. Louis, MO, USA). 5-Hydroxyindole ethanol (5-HTOL), 5-hydroxyindole acetic acid (5-HIAA), and 5-hydroxytryptophan (5-HTP) were purchased from Toronto Research Chemicals (Toronto, Ontario, Canada). Pyridine, and N,O-bis-(trimehylsilyl) trifluoroacetamide, (BSTFA)trimethylchlorosilane (TMCS) were purchased from Sigma also. Cleanert PEP-2 SPE cartridges $(6 \mathrm{~mL} / 200 \mathrm{mg})$ were obtained from Bonna-Agela Technologies (Tianjin, China).

This study was approved by the Human Subjects Committee of Hebei Medical University. CSF specimens were collected from 36 newly diagnosed acute lymphoblastic leukemia (ALL) children without chemotherapy who were admitted to the Department of Pediatrics, Second Affiliated Hospital of Hebei Medical University, between March 2018 and November 2018. There were 20 boys and 16 girls; the boys' ages were 2.4-10.4 years and the median age was 6.2, and the girls' ages were 2.9-9.8 years and the median age was 5.6. There were 12 boys and 12 girls in the control group (CON); the boys' ages were 1.8-10.6 years and the median age was 6.7, and the girls' ages were 2.2-11.4 years and the median age was 7.1. These children had undergone lumbar puncture for virus detection at the same time, and the screened subjects did not suffer from known tumors of the nervous system and infection. There were no statistical differences in gender and age between the two groups, and all the collected CSF specimens were stored at $-80^{\circ} \mathrm{C}$.

The frozen samples were thawed at room temperature, and $3 \mathrm{~mL}$ of fluid from each sample was analyzed. Solid-phase extraction (SPE) with Cleanert PEP-2 column was used to concentrate the target and eliminate the interference, $2 \mathrm{~mL}$ of distilled water was applied to the cartridge in a wash step, and then $1 \mathrm{~mL}$ of acidified methanol ( $0.5 \%$ formic acid) was used to elute the analytes. The eluent was collected and dried with a slow stream of nitrogen at $70{ }^{\circ} \mathrm{C}$. Seventy microliter of N,O-bis-trimethylsilyltrifluoroacetamide-trimethylchlorosilane (BSTFA-TMCS) (99:1, V/V) $+30 \mu \mathrm{L}$ pyridine $+2.5 \mu \mathrm{L}$ methanol agent were used in derivatization, and after that under the condition of $95{ }^{\circ} \mathrm{C}$ at a duration of $60 \mathrm{~min}$ for derivatization, $1 \mu \mathrm{L}$ of each sample was injected into the gas chromatography-mass spectrometry (GC-MS) (Agilent, Little Falls, DE, USA) for analysis. The analysis procedure was the same as described before [8]. For a change, a DB- $530 \mathrm{~m} \times 0.25 \mathrm{~mm}$ I.D., $0.25 \mu \mathrm{m}$ film thickness column (Agilent, Santa Clara, CA, USA) was chosen in order to improve the resolution and sensitivity.

The data are expressed as the mean \pm standard deviation, and were analyzed using SPSS 13.0 software (SPSS
Inc., Chicago, IL, USA). Independent t-tests were subsequently carried out.

\section{Results}

The matrix effect (ME) was verified by comparing the peak areas of the analyte standards dissolved in ultrapure water with the peak areas of analytes in the CSF matrix. According to the concentration of practical samples, five standards of the analytes and five spiked CSF samples were investigated at concentration levels as follows: 5-HTOL, $5.0 \mu \mathrm{g} / \mathrm{L} ; 5$-HIAA, $50.0 \mu \mathrm{g} / \mathrm{L}, 5$-HTP, $5.0 \mu \mathrm{g} / \mathrm{L} ; 5$-HT, 5.0 $\mu \mathrm{g} / \mathrm{L}$. The formula used was: ME (\%) $=$ (Area of analytes in the spiked sample - Area in the unspiked sample) $\times 100$ / (Area in water solution). The results showed that the ME of 5-HTOL, 5-HIAA, 5-HTP and 5-HT was 95.3 $\pm 3.2 \%$, $98.3 \pm 2.4 \%, 92.3 \pm 5.3 \%$ and $106.2 \pm 7.2 \%$, respectively, which indicated that there was no significant ME that could influence the analytes. Hence, the calibration curve was obtained by spiking ultrapure water over the concentration range of $0.5-200.0 \mu \mathrm{g} / \mathrm{L}, 0.5-200.0 \mu \mathrm{g} / \mathrm{L}, 2.0-$ $800.0 \mu \mathrm{g} / \mathrm{L}$ and 2.0-800.0 $\mu \mathrm{g} / \mathrm{L}$ for 5-HTOL, 5-HIAA, 5-HTP and 5-HT, respectively. Favorable linear relationships were achieved in the tested calibration ranges, and the correlation coefficient values $\left(\mathrm{R}^{2}\right)$ of 5-HTOL, 5-HIAA, 5-HTP and 5-HT were 0.9924, 0.9952, 0.9936 and 0.9918, respectively. The method sensitivity was evaluated by determining the limit of detection (LOD) and the limit of quantitation (LOQ) for each compound. The LODs were calculated as the sample concentration that produces a peak with a height 3 times that of the signal-to-noise ratio. The LODs of 5-HTOL, 5-HIAA, 5-HTP and 5-HT were 0.1, 0.1, 0.3 and $0.4 \mu \mathrm{g} / \mathrm{L}$, respectively. The LOQs were established to be 0.5 $\mu \mathrm{g} / \mathrm{L}, 0.5 \mu \mathrm{g} / \mathrm{L}, 2.0 \mu \mathrm{g} / \mathrm{L}$ and $2.0 \mu \mathrm{g} / \mathrm{L}$ for 5-HTOL, 5-HIAA, 5-HTP and 5-HT, respectively, the lowest analyte concentration on the standard curve that could be measured with adequate precision [coefficient of variation (CV) $<20 \%$, $\mathrm{n}=6$ ] [9]. Recovery was measured by spiking known amounts of standards in known CSF samples in triplicate at three concentration levels. The intra-day precision was determined on six replicates in a single day, and the interday precision was determined during 3 days by analyzing replicates of each samples. The related data are shown in Table 1.

Under the optimum condition, we examined the levels of 5-HTOL, 5-HIAA, 5-HTP and 5-HT in CSF from the ALL and CON groups. The typical chromatograms of standard solution, an extract CSF from ALL and an extract CSF from CON are shown in Figure 1. The results 
Table 1: The recovery and coefficient of variation (CV) in CSF of GC-MS analysis.

\begin{tabular}{|c|c|c|c|c|c|c|c|}
\hline \multirow[t]{2}{*}{ Compound } & \multirow[t]{2}{*}{ Concentration, $\mu \mathrm{g} / \mathrm{L}$} & \multicolumn{3}{|c|}{ Intra-day $(n=6)$} & \multicolumn{3}{|c|}{ Inter-day $(n=6)$} \\
\hline & & Mean, $\mu \mathrm{g} / \mathrm{L}$ & Recovery, \% & CV, \% & Mean, $\mu \mathrm{g} / \mathrm{L}$ & Recovery, \% & $\mathrm{CV}, \%$ \\
\hline \multirow[t]{3}{*}{ 5-HTOL } & 5.0 & 5.2 & 104.6 & 1.8 & 5.2 & 104.8 & 2.6 \\
\hline & 25.0 & 25.7 & 102.8 & 1.9 & 26.3 & 105.2 & 2.4 \\
\hline & 100.0 & 103.5 & 103.4 & 2.3 & 103.8 & 103.8 & 1.8 \\
\hline \multirow[t]{3}{*}{ 5-HIAA } & 5.0 & 5.3 & 105.6 & 1.5 & 4.9 & 97.8 & 2.8 \\
\hline & 25.0 & 25.9 & 103.5 & 1.4 & 24.7 & 98.8 & 2.3 \\
\hline & 100.0 & 99.3 & 99.3 & 2.3 & 103.5 & 103.5 & 2.4 \\
\hline \multirow[t]{3}{*}{ 5-HTP } & 20.0 & 19.5 & 97.4 & 3.5 & 18.6 & 93.0 & 4.3 \\
\hline & 100.0 & 95.2 & 95.2 & 3.8 & 95.1 & 95.1 & 4.5 \\
\hline & 400.0 & 378.6 & 94.6 & 4.5 & 384.1 & 96.0 & 3.1 \\
\hline \multirow[t]{3}{*}{$5-\mathrm{HT}$} & 20.0 & 20.5 & 102.6 & 3.6 & 21.2 & 106.1 & 4.2 \\
\hline & 100.0 & 104.3 & 104.3 & 3.0 & 106.9 & 106.9 & 3.7 \\
\hline & 400.0 & 420.5 & 105.1 & 3.7 & 412.2 & 103.1 & 3.0 \\
\hline
\end{tabular}

5-HIAA, 5-hydroxyindole acetic acid; 5-HT, 5-hydroxytryptamine; 5-HTOL, 5-hydroxyindole ethanol; 5-HTP, 5-hydroxytryptophan; CSF, cerebrospinal fluid; CV, coefficient of variation; GC-MS, gas chromatography-mass spectrometry.

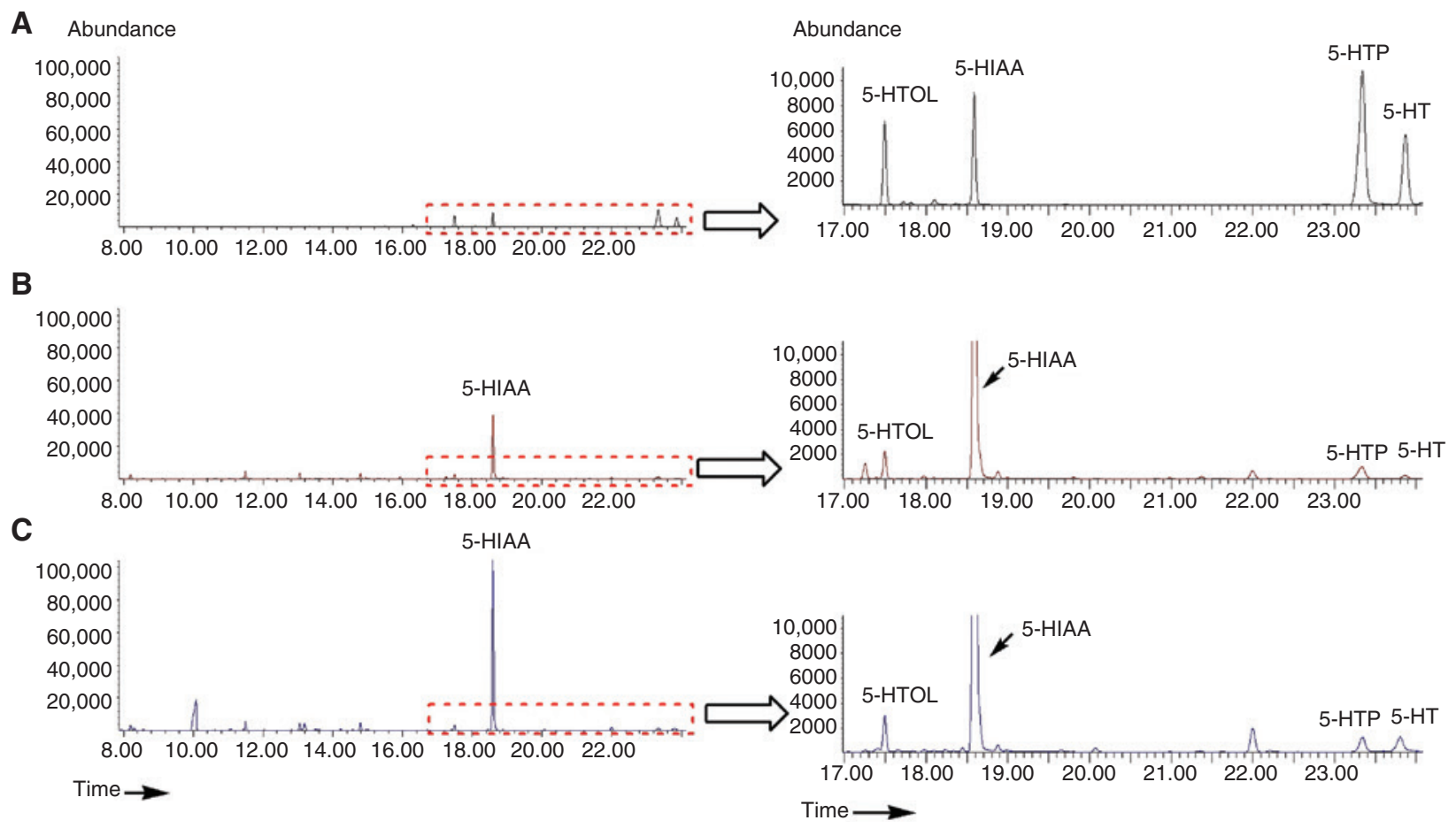

Figure 1: Typical chromatograms of 5-HTOL, 5-HIAA, 5-HTP and 5-HT analysis by the GC-MS method.

(A) Aqueous standard of $10 \mu \mathrm{g} / \mathrm{L} 5-\mathrm{HTOL}, 10 \mu \mathrm{g} / \mathrm{L} 5-\mathrm{HIAA}, 40 \mu \mathrm{g} / \mathrm{L} 5-\mathrm{HTP}$ and $40 \mu \mathrm{g} / \mathrm{L}$ 5-HT, respectively, (B) an extract CSF from ALL, (C) an extract CSF from CON.

showed that the 5-HT and 5-HIAA contents in the ALL group were significantly lower than those in the CON group $(\mathrm{p}<0.01)$, while there was no significant difference in 5-HTP and 5-HTOL contents between the two groups ( $\mathrm{p}>0.05$ ). Figure 2 shows the difference in the excretion levels of 5-HTOL, 5-HIAA, 5-HTP and 5-HT in CSF from the ALL and CON groups.

\section{Discussion}

Our study demonstrates that leukemia may disturb the metabolism of 5-HT and related indole derivatives in the CSF. The contents of 5-HT and 5-HIAA in the ALL group were significantly lower than those in the CON group, possibly owing to the demands of fast-growing leukemic 

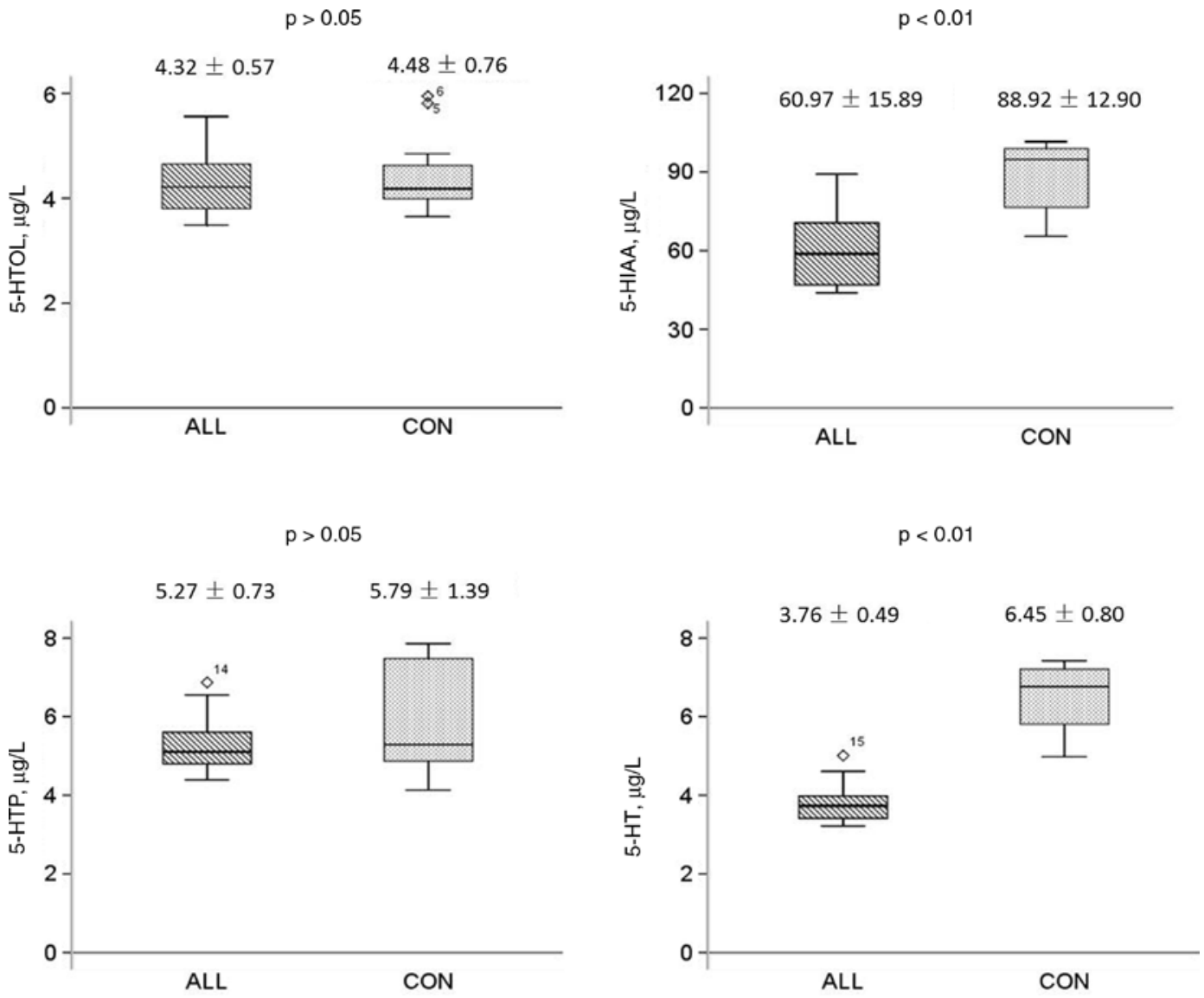

Figure 2: Difference in the excretion levels of 5-HTOL, 5-HIAA, 5-HTP and 5-HT in CSF from the ALL and CON groups.

cells for large amounts of amino acids for protein synthesis. Moreover, leukemia patients are susceptible to infection that may injure the 5-HT neurons and impede the synthesis of 5-HT and 5-HIAA, resulting in insufficient 5-HT and 5-HIAA, and thus reduced levels in the CSF [10]. Reports on the association between the contents of 5-HT/related indole derivatives in the CSF and ALL are limited. More studies are required to reveal the mechanism underlying the role of 5-HT and related indole derivatives in the pathogenesis of ALL. We hope that our study provides a new dimension to the pathology and treatment of ALL.

Author contributions: All the authors have accepted responsibility for the entire content of this submitted manuscript and approved submission.

Research funding: This work was supported by the Natural Science Foundation of Hebei Province (No. H2018206122) and financial from the Department of Health of Hebei province (20180117).

Employment or leadership: None declared.

Honorarium: None declared.
Competing interests: The funding organization(s) played no role in the study design; in the collection, analysis, and interpretation of data; in the writing of the report; or in the decision to submit the report for publication.

\section{References}

1. Hu L, Wang Q, Qin Z, Su K, Huang L, Hu N, et al. Detection of 5-hydroxytryptamine (5-HT) in vitro using a hippocampal neuronal network-based biosensor with extracellular potential analysis of neurons. Biosens Bioelectron 2015;66:572-78.

2. Qiu Y, Cai G, Su M, Chen T, Liu Y, Xu Y, et al. Nutrition. Urinary metabonomic study on colorectal cancer. J Proteome Res 2010;9:1627-34.

3. Yin H, Park SA, Han SK, Park SJ. Effects of 5-hydroxytryptamine on substantia gelatinosa neurons of the trigeminal subnucleus caudalis in immature mice. Brain Res 2011;1356:91-101.

4. Zhang HQ, Zhang Y, Liu L, Li JL, Lu YC, Yu YY, et al. Neural connection supporting endogenous 5-hydroxytryptamine influence on autonomic activity in medial prefrontal cortex. Auton Neurosci 2017;203:25-32.

5. Page KM, Labopin M, Ruggeri A, Michel G, Diaz de Heredia C, O'Brien T, et al. Factors associated with long-term risk of relapse 
after unrelated cord blood transplantation in children with acute lymphoblastic leukemia in remission. Biol Blood Marrow Transplant 2017;23:1350-8.

6. Sokomba EN, Patsalos PN, Lolin YI, Curzon G. Concurrent monitoring of central carbamazepine and transmitter amine metabolism and motor activity in individual unrestrained rats using repetitive withdrawal of cerebrospinal fluid. Neuropharmacology 1988;27:409-15.

7. Ogawa S, Tsuchimine S, Kunugi H. Cerebrospinal fluid monoamine metabolite concentrations in depressive disorder: a meta-analysis of historic evidence. J. Psychiatr Res 2018;105:137-46.
8. Shi HM, Wang B, Niu Lm, Cao MS, Kang WJ, Lian KQ, et al. Trace level determination of 5 -hydroxytryptamine and its related indoles in amniotic fluid by gas chromatography-mass spectrometry. J Pharmaceut Biomed 2017;143:176-82.

9. Margalho C, Castanheira A, Reala FC, Gallardo E, López-Rivadulla M. Determination of "new psychoactive substances" in postmortem matrices using microwave derivatization and gas chromatography-mass spectrometry. J Chromatogr B 2016;1020:14-23.

10. Papiez MA, Dybala M, Sowa-Kucma M, Krzysciak W, Taha H, Jozkowicz A, et al. Evaluation of oxidative status and depression-like responses in Brown Norway rats with acute myeloid leukemia. Prog Neuro Biol Psychoph 2009;33:596-604. 